\title{
Prevention and Management of Dental Trauma in Primary Teeth in the Context of the COVID-19: A Critical Literature Review
}

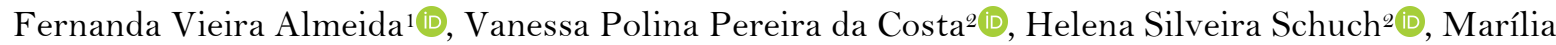 \\ Leão Goettems 2 (1)
}

'School of Dentistry, Federal University of Pelotas, Pelotas, RS, Brazil.

${ }^{2}$ Graduate Program in Dentistry, Federal University of Pelotas, Pelotas, RS, Brazil.

Correspondence: Marília Leão Goettems, Graduate Program in Dentistry, Federal University of Pelotas, Rua Gonçalves Chaves, 457, Pelotas, RS, Brazil. 96015-560. E-mail: marilia.goettems@gmail.com

Academic Editor: Alidianne Fábia Cabral Cavalcanti

Received: 09 April 2021 / Review: 29 May 2021 / Accepted: 25 July 2021

\begin{abstract}
How to cite: Almeida FV, Costa VPP, Schuch HS, Goettems ML. Prevention and management of dental trauma in primary teeth in the context of the COVID-19: a critical literature review. Pesqui Bras Odontopediatria Clín Integr. 2021; 21:e2 10077. https://doi.org/10.1590/pboci.2021.161
\end{abstract}

\begin{abstract}
Objective: To evaluate and discuss the prevention and management of dental trauma in primary teeth during the COVID-19 pandemic. Material and Methods: Critic literature review, searching electronic databases and Google for articles and guidelines published in English that described prevention and management of dental trauma in primary dentition during the pandemic of COVID-19. Results: 5 publications regarding management and 1 leaflet about prevention for parents were identified. Urgent care is required when the child presents tooth fracture resulting in pain or causing soft tissue trauma and luxation affecting bite. Avulsed teeth should not be replanted and advice and self-help may be sufficient in some situations. Instructions about soft diet and hygiene must be provided. Remote consults are recommended for non-urgent situations and during follow-up to evaluate the presence of sequelae. Aerosol generating procedures should be avoided and, in cases of poor prognosis, extraction is recommended to prevent recurring visits to the dental office. Conclusion: During COVID-19 pandemic, remote consults should be recommended to evaluate traumatic dental injuries in primary dentition. Follow-up should not be neglected and may be performed through remote consultation.

Keywords: Pediatric Dentistry; COVID-19; Tooth Injuries; Emergencies.
\end{abstract}




\section{Introduction}

Traumatic dental injury (TDI) is an accidental or intentional impact injury to the teeth and/or supporting tissues. Due to its nature, it cannot be expected and often requires emergency attention [1]. It can occur at all ages, but the majority of TDI takes place in childhood [2]. Reviews of the literature suggest that from one-fourth [3] to one-third [4] of children in primary dentition age experience TDIs. This problem may be distressful for parents and children and have physical, aesthetical and psychological consequences [2]. Dental trauma is often related to falls, sports, and collisions with objects and people, all of which are common events in children's daily routine. The main place where TDI occurs is at home, followed by preschool/school and outdoors [5].

At the beginning of 2020, the world witnessed the development and spread of a new virus, the SarsCoV-2 (severe acute respiratory syndrome coronavirus 2 ), the coronavirus that causes the coronavirus disease 2019 (COVID-19). In March 2020, the World Health Organization declared the COVID-19 as a pandemic disease [6]. One of the most consistent interventions to stop the spread of Sars-CoV-2 worldwide is the closure of schools [7]. The proportion of countries adopting the measure varied during the different phases of the disease. Still, the country-wide closures took place in as much as 193 countries at the same time, affecting more than $90 \%$ of the total enrolled learners worldwide (from pre-primary to tertiary education students) [8]. Data from the United Nations Educational, Scientific and Cultural Organization [8] show that, at the end of September 2020, still almost 50\% of the total enrolled learners were affected by the measure. The school closures mean children are spending most of their time at home, and virtual school lessons are a reality in many countries. Also, as another measure to fight COVID-19, governments recommend people avoid public places and practice social distancing. All these measures reflect on children and families staying in restricted physical spaces, usually inside the house, which may predispose the occurrence of domestic accidents. In fact, domestic accidents in children have been pointed as potential collateral damage of the COVID-19 pandemic [9]. One of the possible consequences of domestic accidents is TDIs.

The treatment for TDIs is a two-step process: immediate emergency care and follow-up. Proper care during the whole treatment course is of paramount importance to minimize the risk of immediate and longterm unfavourable outcomes [10]. In the short-term, dental trauma management is essential to stop the pain, restore oral function and aesthetics, and reduce dental anxiety, and avoid infections [11]. Severe TDI can present a sequel in the affected teeth even after 3-4 years of follow-up [12] and also affect the permanent successors [13]. There are recently published and internationally recognized guidelines from the International Association of Dental Traumatology on the management of TDI to the primary teeth [11]. However, the COVID-19 pandemic affects health care provision significantly, and the standard recommended care is not always feasible to be provided. Considering the high risk of transmission of COVID-19 in oral health care settings, the WHO guided professionals to provide only essential oral health services in the context of the COVID-19 [14]. The management of dental/orofacial trauma is among the urgent/emergency interventions listed that should be provided [15].

The management of dental trauma is complex and even more challenging in primary or early mixed dentition [16]. Immediate management and follow-up of TDIs are vital to minimize the impacts of this problem on the child's life. However, many dentists are unsure how to proceed and, in the context of the COVID-19 pandemic, are afraid of being infected or passing the SARS-CoV-2 infection to patients. Therefore, this review aims to identify scientific productions developed on the prevention and management of traumatic dental injuries to the primary teeth during the COVID-19 pandemic. 


\section{Material and Methods}

An integrative literature review was conducted [17] to answer the following research question: "what are the recommendations for the prevention and management of traumatic dental injuries to the primary teeth during the COVID-19 pandemic?”.

\section{Search Strategy and Data Collection}

An electronic search was conducted in PubMed, SciELO, Google Scholar and Web of Science databases without language restrictions to identify articles published up to September 2020. Search strategy as run in each database is showed in Chart 1.

\section{Chart 1. Search strategy.}

\begin{tabular}{|c|c|}
\hline Database & Search Strategy \\
\hline PubMed & 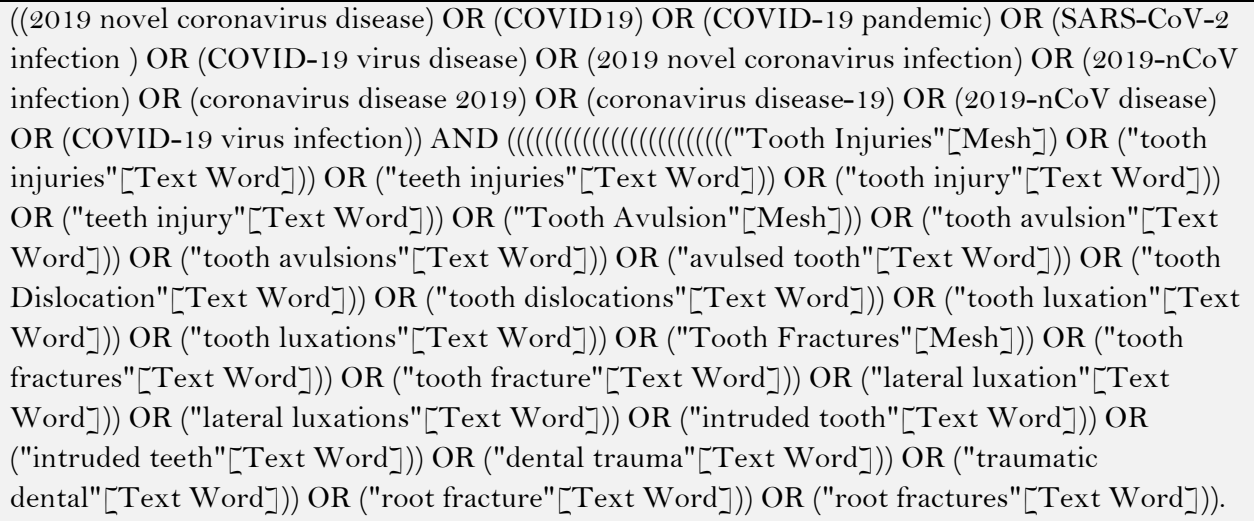 \\
\hline SciELO & $\begin{array}{l}\text { "dental trauma" OR "traumatic dental injuries" OR "tooth injuries" OR "teeth injuries" OR } \\
\text { "tooth injury" OR "teeth injury" OR "tooth avulsion" OR "tooth avulsions" OR "avulsed tooth" } \\
\text { OR "tooth dislocation" OR "tooth dislocations" OR "tooth luxation" OR "tooth luxations" OR } \\
\text { "Tooth Fractures" OR "tooth fracture" OR "lateral luxation" OR "lateral luxations" OR } \\
\text { "intruded tooth" OR "intruded teeth" OR "root fractures") AND ((2019 novel coronavirus } \\
\text { disease) OR (COVID19) OR (COVID-19 pandemic) OR (SARS-CoV-2 infection) OR (COVID-19 } \\
\text { virus disease) OR (2019 novel coronavirus infection) OR (2019-nCoV infection) OR (coronavirus } \\
\text { disease 2019) OR (coronavirus disease-19) OR (2019-nCoV disease) OR (COVID-19 virus } \\
\text { infection)) }\end{array}$ \\
\hline Web of Science & $\begin{array}{l}\text { ((2019 novel coronavirus disease) OR (COVID 19) OR (COVID-19 pandemic) OR (SARS-CoV-2 } \\
\text { infection ) OR (COVID-19 virus disease) OR (2019 novel coronavirus infection) OR (2019-nCoV } \\
\text { infection) OR (coronavirus disease 2019) OR (coronavirus disease-19) OR (2019-nCoV disease) } \\
\text { OR (COVID-19 virus infection)) AND ("Tooth Injuries") OR ("teeth injuries") OR ("tooth } \\
\text { injury") OR ("teeth injury") OR ("Tooth Avulsion") OR ("tooth avulsions") OR ("avulsed tooth") } \\
\text { OR ("tooth Dislocation") OR ("tooth dislocations") OR ("tooth luxation") OR ("tooth luxations") } \\
\text { OR ("Tooth Fractures") OR ("tooth fracture") OR ("lateral luxation") OR ("lateral luxations") } \\
\text { OR ("intruded tooth") OR ("intruded teeth") OR ("dental trauma") OR ("traumatic dental") OR } \\
\text { ("root fracture") OR ("root fractures")) }\end{array}$ \\
\hline
\end{tabular}

\section{Eligibility Criteria}

Were considered eligible for this study bibliographic review, systematic reviews, meta-analyses, randomized controlled trials, cohort studies and guidelines or recommendations from official associations, such as the British Society of Paediatric Dentistry and the International Association of Dental Traumatology. Publications in English, Italian, Spanish, and Portuguese were considered.

The exclusion criteria included articles not related to the topic, animal studies, manuscripts only available under subscription, and articles in languages other than the aforementioned. No time limits were applied during the screening phase of the scientific articles. 


\section{Results}

The search strategy identified seven articles in PubMed, 1 in SciELO, and 5 in Web of Science. None of these articles described management and prevention of traumatic dental injuries in primary teeth during COVID-19. One manuscript describing a brief management protocol for dentoalveolar trauma was found, but with no recommendations for primary dentition [18]. One article described the operating procedure of a hospital [19]. From Google, 4 publications from official dental associations regarding the management of TDI in the context of the COVID-19 and 1 leaflet about prevention for parents [20] were identified. The flow diagram of the study selection is presented in Figure 1.

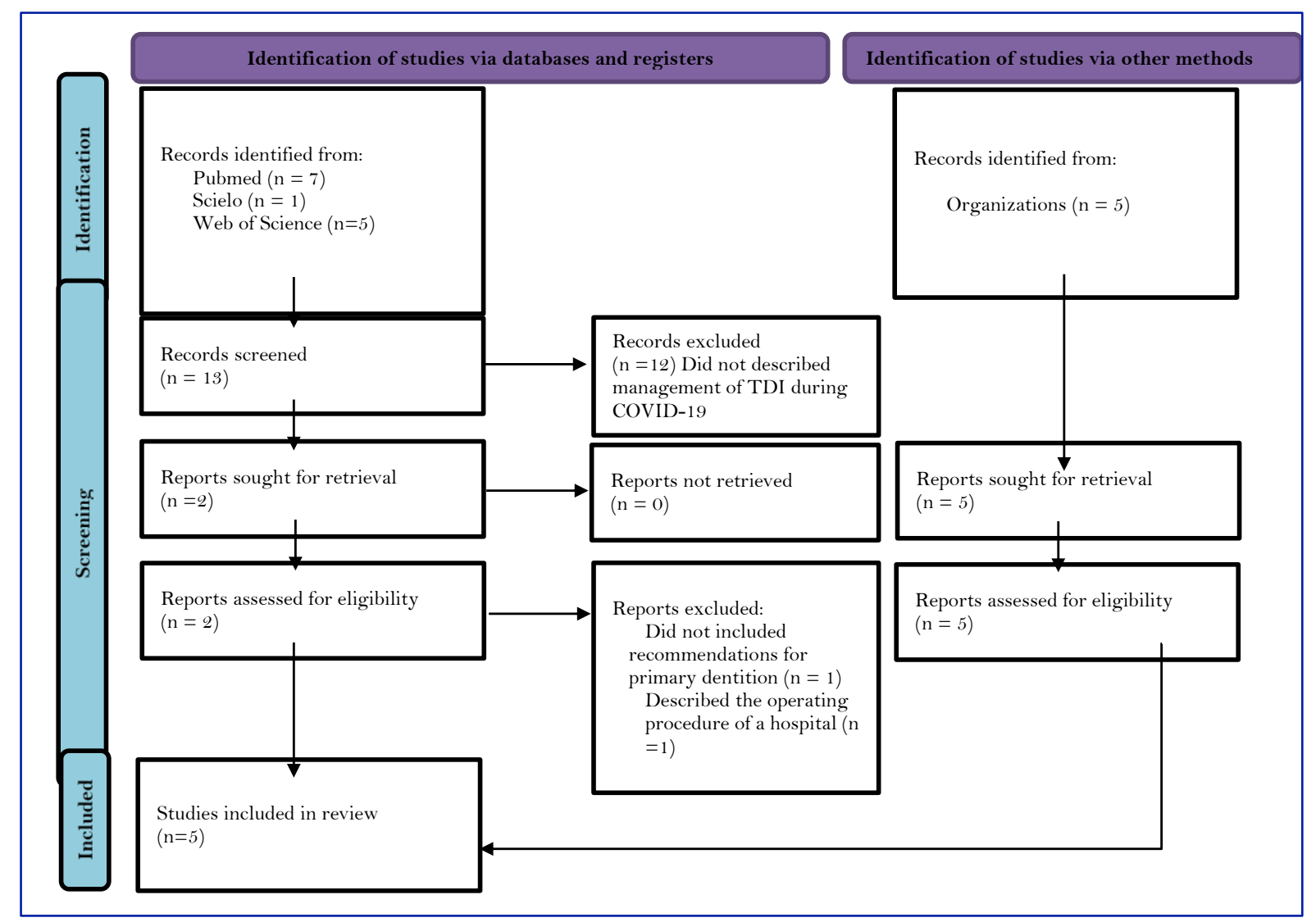

Figure 1. Flow diagram of study selection.

Prevention of Traumatic Dental Injuries

To prevent oral diseases during the COVID-19 pandemic, The International Association of Paediatric Dentistry (IAPD) [20] presented one series of infographics to support families during the lockdown called "Stay Home, Stay Healthy". One of the infographics offers tips about prevention of dental trauma at home, including: 1. Avoid running in the house; 2. Avoid jumping on the bed/sofa; 3. Avoid throwing toys/objects; and 4. Avoid placing toys/objects in the mouth.

Due to the pandemic, some dental procedures are not being performed because they are considered elective and urgent care is being prioritized. In this scenario, it is crucial to prioritise and emphasise all oral health prevention and education [21,22]. Families are spending most of the time at home, following social distancing recommendations. The home setting can stimulate more lively play modes in the children, with an increased risk of traumatic events affecting the dental elements and the consequent need for urgent interventions that increase the risk of contagion from COVID-19 [22]. 
The home environment plays an important part in the occurrence of injuries, particularly falls. A relation seems to exist between the number of injury hazards in the home and the risk of home injury, although many factors could potentially confound this association [23]. Some studies have already shown that, depending on the age group, the home is the place where trauma occurs most frequently [24]. Babies, children, and elderly are the categories most at risk for domestic accidents [25]. Early childhood, when the baby starts to walk, is the most frequent moment of dental trauma occurrence. Therefore, parents should be instructed on the need for careful supervision of the child.

Interventions designed to reduce home injuries have focused on the removal of a particular hazard or intensive broad educational strategies [26]. One of the major public health strategies to prevent child injuries at home is by promoting safety practices among parents [27]. During the pandemic, it is of utmost importance that educational strategies to prevent home injuries be promoted.

Home safety education and the provision of safety equipment can be effective in increasing some, but not all, safety practices, including safety equipment possession. There is some evidence that home safety interventions may be useful in reducing rates of all injuries combined. To falls preventions some measures can be taken as: Fitted stair gates, avoid possession and use of a baby walker, possession of window locks or screens or windows with a limited opening, possession of non-slip bath mats or decals, does not leave a child alone on a high surface [28].

A study compared the changes in the characteristics of patients treated for TDIs before and during the transmission control measures in Wuhan City, China, and found that the proportion of patients in different age groups changed considerably. In 2020, the high proportion of fall injuries was a remarkable finding, and children aged 0 to 6 were the majority of patients. The possible explanation is that children stayed at home with limited living space, and their self-protection awareness is worse than that of adults. This led to an increased risk of TDIs in children aged o to 6. In addition, the aetiology of TDIs was closely related to the age of the patient [29].

Oral trauma in infants can also be caused by animal bites (mainly cats and dogs). Prevention strategies against dog bites should include careful supervision of the interaction between infants and dogs, education relating to the responsibility of dog owners and observance of the rules relating to the control of animals [25].

It is paramount to remember that our responsibilities to safeguard children and young people continue during the pandemic. One should be aware that the patients may be exposed to increased risks of abuse or neglect during the lockdown, particularly of witnessing domestic abuse [30].

\section{Management of Dental Trauma in Primary Dentition during COVID-19 Pandemic}

In August $3^{\text {rd }}$, 2020, the World Health Organization published Interim guidance for the provision of essential oral health services during the COVID-19 pandemic, in which they advise that routine non-essential oral health care - which usually includes oral health check-ups, dental cleanings and preventive care - be delayed until there has been sufficient reduction in COVID-19 transmission rates from community transmission to cluster cases or according to official recommendations at national, sub-national or local level [14]. The main reason is that oral health care teams work close to patients' faces for prolonged periods. Their procedures involve face-to-face communication and frequent exposure to saliva, blood, and other body fluids and handling sharp instruments - consequently, dentists are at high risk of being infected with SARS-CoV-2 or passing the infection to patients WHO. However, urgent or emergency oral health care interventions that are 
vital for preserving a person's oral functioning, managing severe pain, or securing quality of life should be provided, including dental trauma.

Traumatic dental injuries may require immediate care and long-term follow-up. Clinical protocols have been developed by institutional agencies linked to health and dentistry, prioritizing the safety and wellbeing of professionals and patients. Dentists must evaluate when face-to-face consultation is required for urgent care or to treat sequels and when advice through remote care is sufficient, especially considering that monitoring only is usually the first treatment choice in the primary dentition. Bellow, the published articles and guidelines are described according to the publication date, and a recommendation about TDI management is summarized.

In March 2020, the Scottish Dental Clinical Effectiveness Programme published a guide about the management of acute dental problems during the COVID-19 pandemic [31]. It recommends that mild and moderate symptoms may be managed remotely by general dental practitioners providing advice and self-help, which might involve analgesics and antimicrobials. Urgent care is required for severe or uncontrolled symptoms that cannot be managed by the patient and require the patient to see a dentist in a designated urgent dental care centre. In the case of dentoalveolar injuries, when the patient does not require emergency medical attention, they should be advised to: clean the affected area by rinsing it gently with mild antiseptic and, if a foreign object(s) is present in the mouth, to remove them; to apply ice packs to soft tissue injury and swelling; to apply pressure with a finger to stop any bleeding. The dentist may consider recommending analgesia. For avulsed, displaced or fractured teeth, besides the recommendations, urgent care is recommended if a primary tooth (or teeth) has been moved out of its usual position and is affecting the bite. In the case of a primary tooth knocked out, considering that primary teeth should not be re-implanted, advice and self-help are the recommendation and should include appropriate analgesia and a soft diet. Also, if a primary tooth (or teeth) has been displaced without affecting the bite, no urgent care is recommended, and dentist should advise the parent/carer to alter the child's diet to include soft food and analgesia if required.

The British Society of Paediatric Dentistry (BSPD) has published consensus guidelines on the management of dental trauma concerning the COVID-19 pandemic, including instructions about primary dentition acute management of traumatic injuries and follow-up care. Remote consults are recommended during follow-up to assess oral hygiene, healing, and complications. If deemed necessary, the patient is seen in the clinic for detailed assessment and treatment. In case of root fracture, all the follow-up could be made through remote consults, 1 week after TDI, 4 weeks and one year after. For enamel-dentine fractures, the follow-up could also be remote, and the recommended time is 8 weeks.

Regarding supporting tissues injuries, even in cases of lateral luxation and intrusion, which have higher risks of sequels and require more frequent follow-ups for monitoring possible alterations, authors recommend that remote consult be performed. Follow-up should include 1-week remote consult for all supporting tissue trauma and, depending on the type of trauma, follow-up at 8 weeks, 6 months and 1 year are required. Post-injury advice should be provided, including care when eating not to further traumatize the injured teeth while encouraging a return to normal function as soon as possible; cleaning the affected area with a soft brush or cotton swab combined with an alcohol-free chlorhexidine gluconate mouth rinse applied topically twice a day for one week to encourage gingival healing and prevent plaque accumulation.

The King College Hospital has published new standard operating procedures specifically for the COVID-19 pandemic, published by Ilyas et al. in June 2020 [19]. According to this guideline, primary tooth trauma standard operating procedure includes telephone advice only or a dental visit to perform an extraction. 
Telephone advice only is given for all cases of enamel/enamel-dentine fracture, concussion, subluxation, intrusion, and extrusion/lateral luxation when not interfering with occlusion/airway risk. Only enameldentine fractures and pathological mobility, causing occlusal inference/airway risk should be seen urgently for treatment.

The Royal College of Surgeons of England published the recommendations for Paediatric Dentistry during COVID-19 pandemic of COVID-19 [30]. These guidelines are intended for use by dental teams working in England and state that no routine dentistry should be provided for children during this pandemic. Routine Dentistry should be deferred to minimise risk to patients, staff, and the public. Traumatic dental injuries to the primary dentition resulting in pulp exposure or severe luxation such that tooth mobility constitutes a potential airway risk and/or is severely interfering with occlusion/function require urgent paediatric dental care. According to the protocol, an extraction may be the preferred treatment option for children with pulpal symptoms to reduce the need for aerosol-generating procedures (AGPs). In June 2020, recommendations for Paediatric Dentistry during the recovery phase of the COVID-19 pandemic, that is, when pandemic activity appeared to be decreasing, were published [32]. Clinical urgency must take priority over a referral to treat (RTT) times. Whilst the latter remains relevant, clinical teams must be able to prioritize care based on clinical urgency (including well-being and quality of life factors). The aim of trauma management should be to minimise the number of visits required and the number of AGPs provided. Outcomes should be optimised by giving evidence-based initial management with a low risk of complications requiring further intervention.

Suppose it is determined that emergency face-to-face dental care is necessary. In that case, anamnesis should be reinforced: before scheduling the emergency treatment appointment, COVID-19 screening should be performed due to the asymptomatic incubation period. It is noteworthy that children tend to have fewer symptoms than adults. Therefore all paediatric patients should be considered as potential carriers unless proven otherwise [33].

Regarding treatment options, although conservative treatments are usually recommended (i.e., pulpotomy, composite build-ups, root canal treatment and repositioning and splinting), during the COVID-19 pandemic BSPD and Dental Trauma UK suggest treatment options should focus on either observation or extraction. The same is observed in the King's College Hospital protocol. A more definitive treatment, such as tooth extraction, may avoid recurring visits to the dental office.

\section{Discussion}

The current pandemic has the potential to change the dental landscape for the foreseeable future. Consideration needs to be given to potential vaccine development, the likelihood of a 'second peak' and the ever-changing evidence-based guidelines, which highlight the impact COVID-19 is having upon both dental practitioners and patients. As a result, the long-term practice may be changed indefinitely [19].

The impact of COVID-19 on dentistry has been profound due to how the virus is spread through aerosol [19]. It may be noted that with reopening of dental practices, some recommendations are that AGPs should be avoided if possible and intervention kept to a minimum. Contemporary, minimally invasive procedures that minimise or eliminate aerosol generation should be employed where intervention is indicated throughout the pandemic, and in the future when practice restrictions ease [33].

It is important to consider that the decision to postpone routine dental care during the COVID-19 pandemic will inevitably create a considerable backlog of incompletely treated dental disease in the child 
population as a whole. Health issues in the oral cavity will most certainly occur due to the lack of regular check-ups and care. In addition, caries and periodontal disease will appear more frequently and, regarding trauma, sequelae to primary and permanent successors may go unnoticed without routine dental care and increase the demand for dental services in the future.

Also, the new biosafety recommendations for clinical dental practice may increase the costs for dental consultations and reduce the number of dental consultations per period. For public health services, there will be a reduction in resoluteness, being a challenge for managers to organize the new demand.

Beginning a road to recovery will require a new way of thinking. This will not be a 'return to normal'. As part of this recovery, clinicians will need to completely re-evaluate how services are prioritised and delivered. Relieving children of pain must take priority over routine dental care services [32].

Given the unprecedented nature of the current global pandemic, it is essential that oral health conditions, including the follow-up of dental trauma, do not be neglected. Dental trauma should be managed safely and efficiently, as well as new measures which can help reduce transmission of the virus be adopted. Patients who have been followed due to TDI should be continuously monitored to immediately detect and treat possible complications.

\section{Conclusion}

During COVID-19 pandemic, remote consults should be recommended to evaluate TDIs in the primary dentition. Urgent care is required when the child presents tooth fracture resulting in pain or causing soft tissue trauma and luxation affecting bite, but advice and self-help may be sufficient in some situations. Aerosol generating procedures should be avoided and, in cases of poor prognosis, extraction is recommended to prevent recurring visits to the dental office. Follow-up of TDIs should not be neglected during the pandemic to avoid sequels and may be performed through remote consultation.

\section{Authors' Contributions}

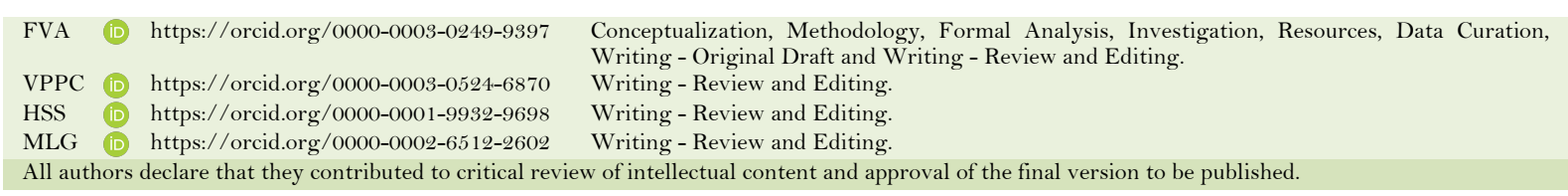

\section{Financial Support}

None.

\section{Conflict of Interest}

The authors declare no conflicts of interest.

\section{Data Availability}

The data used to support the findings of this study can be made available upon request to the corresponding author.

\section{References}

[1] Lam R. Epidemiology and outcomes of traumatic dental injuries: A review of the literature. Aust Dent J 2016; 61(Suppl 1):4-20. https://doi.org/10.1111/adj.12395

[2] Levin L, Day PF, Hicks L, O'Conell A, Fouad AF, Bourguignon C, et al. International Association of Dental Traumatology guidelines for the management of traumatic dental injuries: General introduction. Dent Traumatol 2020; 36(4):309-13. https://doi.org/10.1111/edt.12574 
[3] Petti S, Glendor U, Andersson L. World traumatic dental injury prevalence and incidence, a meta-analysis — One billion living people have had traumatic dental injuries. Dent Traumatol 2018; 34(2):71-8. https://doi.org/10.1111/edt.12389

[4] Glendor U. Epidemiology of traumatic dental injuries - A 12 year review of the literature. Dent Traumatol 2008; 24(6):603-1. https://doi.org/10.1111/j.1600-9657.2008.00696.x

[5] Siqueira MBLD, Gomes MC, Oliveira AC, Martins CC, Granville-Garcia AF, Paiva SM. Predisposing factors for traumatic dental injury in primary teeth and seeking of post-trauma care. Braz Dent J 2013; 24(6):647-54. https://doi.org/10.1590/0103-6440201302352

[6] World Health Organization. Director-General's opening remarks at the media briefing on COVID-19 - 11 March 2020. 2020. Available from: https://www.who.int/director-general/speeches/detail/who-director-general-s-openingremarks-at-the-media-briefing-on-covid-19---11-march-2020. [Accessed on October 18, 2021].

[7] Donohue JM, Miller E. COVID-19 and School Closures. JAMA 2020; 324(9):845-847. https://doi.org/10.1001/jama.2020.13092.

[8] UNESCO. COVID-19 Impact on Education. Available from: https://en.unesco.org/covid19/educationresponse. [Accessed on October 18th, 2021]

[9] Bressan S, Gallo E, Tirelli F, Gregori D, Da Dalt L. Lockdown: more domestic accidents than COVID-19 in children. Arch Dis Child 2020; 106(2):e3. https://doi.org/10.1136/archdischild-2020-319547

[10] Goswami M, Rahman B, Singh S. Outcomes of luxation injuries to primary teeth-a systematic review. J Oral Biol Craniofacial Res 2020; 10(2):227-32. https://doi.org/10.1016/j.jobcr.2019.12.001

[11] Day PF, Flores MT, O'Connell AC, Abbott PV, Tsilingaridis G, Fouad AF, et al. International Association of Dental Traumatology guidelines for the management of traumatic dental injuries: 3. Injuries in the primary dentition. Dent Traumatol 2020; 36(4):343-59. https://doi.org/10.1111/edt.12576

[12] Qassem A, Martins N da M, da Costa VPP, Torriani DD, Pappen FG. Long-term clinical and radiographic follow up of subluxated and intruded maxillary primary anterior teeth. Dent Traumatol 2015; 31(1):57-61. https://doi.org/10.1111/edt.12135

[13] Ranka M, Dhaliwal H, Albadri S, Brown C. Trauma to the primary dentition and its sequelae. Dent Update 2013; 4O(7):534-6, 539-40, 42. https://doi.org/10.12968/denu.2013.40.7.534

[14] World Health Organization. Considerations for the provision of essential oral health services in the context of COVID-19. 2020. Available from: https://www.who.int/publications/i/item/who-2019-nCoV-oral-health-2020.1 [Accessed on October 18, 2021].

[15] Luzzi V, Ierardo G, Bossù M, Polimeni A. Paediatric oral health during and after the COVID-19 Pandemic. Int J Paediatr Dent 2020; 31(1):20-6. https://doi.org/10.1111/ipd.12737

[16] Needleman HL. The art and science of managing traumatic injuries to primary teeth. Dent Traumatol 2011; 27(4):295-9. https://doi.org/10.1111/j.1600-9657.2011.01005.x

[17] Torraco RJ. Writing integrative literature reviews: guidelines and examples. Hum Resour Dev Rev 2005; 4(3):35667. https://doi.org/10.1177/1534484305278283

[18] Ayub K, Alani A. Acute endodontic and dental trauma provision during the COVID-19 crisis. Br Dent J 2020; 229(3):169-175. https://doi.org/10.1038/s41415-020-1920-0.

[19] Ilyas N, Agel M, Mitchell J, Sood S. COVID-19 pandemic: the first wave - an audit and guidance for paediatric dentistry. Br Dent J 2020; 228(12):927-31. https://doi.org/10.1038/s41415-020-1702-8

[20] International Association of Paediatric Dentistry. Infographics. Available from: https://iapdworld.org/education/iapd-infographics/. [Accessed on September 24, 2020].

[21] Al-Halabi M, Salami A, Alnuaimi E, Kowash M, Hussein I. Assessment of paediatric dental guidelines and caries management alternatives in the post COVID-19 period. A critical review and clinical recommendations. Eur Arch Paediatr Dent 2020; 21(5):543-56. https://doi.org/10.1007/s40368-020-00547-5.

[22] Luzzi V, Ierardo G, Bossù M, Polimeni A. COVID-19: pediatric oral health during and after the pandemics. Appl Sci 2020; 2020040002. https://doi.org/10.20944/preprints202004.0002.v 1

[23] Keall MD, Baker M, Howden-Chapman P, Cunningham M. Association between the number of home injury hazards and home injury. Accid Anal Prev 2008; 4O(3):887-93. https://doi.org/10.1016/j.aap.2007.10.003

[24] Costa VPP, Bertoldi AD, Baldissera EZ, Goettems ML, Correa MB, Torriani DD. Traumatic dental injuries in primary teeth: Severity and related factors observed at a specialist treatment centre in Brazil. Eur Arch Paediatr Dent 2014; 15(2):83-8. https://doi.org/10.1007/s40368-013-0068-x

[25] Cagetti MG, Marcoli PA, Berengo M, Cascone P, Cordone L, Defabianis P, et al. Italian guidelines for the prevention and management of dental trauma in children. Ital J Pediatr. 2019; 45(1):157. https://doi.org/10.1186/s13052-019-0734-7

[26] LeBlanc JC, Pless IB, King WJ, Bawden H, Bernard-Bonnin AC, Klassen T, et al. Home safety measures and the risk of unintentional injury among young children: A multicentre case-control study. CMAJ 2006; 175(8):883-7. https://doi.org/10.1503/cmaj.050592 
[27] Peden M, Oyegbite K, Ozanne-Smith J, et al. (Editors). World Report on Child Injury Prevention. Geneva: World Health Organization; 2008. Available from: https://www.ncbi.nlm.nih.gov/books/NBK310641/. [Accessed on October 18, 2021].

[28] Kendrick D, Young B, Mason-Jones AJ, Ilyas N, Achana FA, Cooper NJ, et al. Home safety education and provision of safety equipment for injury prevention (Review). Evidence-Based Child Heal 2013; 8(3):761. https://doi.org/10.1002/ebch.1911

[29] Yang YT, Zhang W, Xie L, Li ZB, Li Z. Characteristic changes of traumatic dental injuries in a teaching hospital of Wuhan under transmission control measures during the COVID-19 epidemic. Dent Traumatol 2020; 36(6):584-9. https://doi.org/10.1111/edt.12589

[30] Royal College of Surgeons. Recommendations for Paediatric Dentistry during COVID-19 pandemic. 2020. Available from: www.rcseng.ac.uk/dental-faculties/fds/coronavirus/. [Accessed on April 04, 2021].

[31] Scottish Dental Clinical Effectiveness Programme. Management of Acute Dental Problems During COVID-19 Pandemic. 2020. Available form: https://www.sdcep.org.uk/published-guidance/acute-dental-problems-covid-19/ [Accessed on October 18, 2021].

[32] Royal College of Surgeons. Recommendations for Paediatric Dentistry during the recovery phase of the COVID-19 pandemic. 2020. Available from: https://www.rcseng.ac.uk/dental-faculties/fds/coronavirus/. [Accessed on April 06, 2021].

[33] Mallineni SK, Innes NP, Raggio DP, Araujo MP, Robertson MD, Jayaraman J. Coronavirus disease (COVID-19): Characteristics in children and considerations for dentists providing their care. Int J Paediatr Dent 2020; 30(3):24550. https://doi.org/10.1111/ipd.12653 